\title{
Plasmon-Enhanced-Attosecond-Extreme Ultraviolet Source
}

\author{
Mattia Lupetti, ${ }^{1, *}$ Matthias F. Kling, ${ }^{2,3}$ and Armin Scrinzi ${ }^{1, \dagger}$ \\ ${ }^{1}$ Physics Department, Ludwig Maximilians Universität, D-80333 Munich, Germany \\ ${ }^{2}$ Max-Planck-Institut für Quantenoptik, D-85748 Garching, Germany \\ ${ }^{3}$ J. R. Macdonald Laboratory, Physics Department, Kansas State University, Manhattan, Kansas 66506, USA
}

(Received 28 November 2012; published 31 May 2013)

\begin{abstract}
A compact high repetition rate attosecond light source based on a standard laser oscillator combined with plasmonic enhancement is analyzed. At repetition rates of tens of $\mathrm{MHz}$, we predict focusable pulses with durations of $\lesssim 300$ as and a spherical wave front at collimation angles $\lesssim 5^{\circ}$. Plasmonic mode and guiding of the attosecond radiation determine the beam parameters. The beam is robust with respect to variations of driver pulse focus and duration.
\end{abstract}

DOI: 10.1103/PhysRevLett.110.223903

PACS numbers: 42.65.Re, 42.72.Bj

High harmonic sources with controlled attosecond $\left(1\right.$ as $\left.=10^{-18} \mathrm{~s}\right)$ time structure allow the observation of electronic dynamics on the natural time scale of valence electrons. A range of recently developed techniques for ultrafast spectroscopy rely on such sources, for example, attosecond streaking of photoemission from atoms [1,2], and solid surfaces [3], as well as attosecond transient absorption spectroscopy [4].

The attosecond pulse sources used in present experiments are all based on high harmonic emission from gases. An extremely nonlinear conversion processingeniously described by the classical three-step recollision model [5,6]-imprints the time structure of the driving laser pulse onto the harmonic radiation. Nonlinearity sharpens the original sinelike driver electric field to produce bursts of high frequency radiation which may be as short as 67 as [7]. The bursts are time delayed relative to the maxima of the driver field by $\sim 0.2$ driver optical cycles; i.e., emission occurs near the nodes of the driver field. The time locking of the attosecond pulse to the driver is exploited for precision time-delay experiments with time resolutions down to $\sim 10$ as.

Further key parameters of the sources are photon energy, pulse separation, peak and average intensity, and for certain applications [8], focal spot size. Maximal photon energies of high gas harmonics depend on driver pulse intensity as $\sim I_{p}+3.2 U_{p}$, where $I_{p}$ is the ionization potential of the gas and $U_{p}$ is the driver ponderomotive potential. This dependency is a direct consequence of the recollision mechanism. For photon energies $\gtrsim 40 \mathrm{eV}$ with pulses at the Ti:sapphire wavelength of $800 \mathrm{~nm}$, one needs intensities $\gtrsim 10^{14} \mathrm{~W} / \mathrm{cm}^{2}$. At driver pulse durations of $\sim 20 \mathrm{fs}$ a short "train" of attosecond pulses is generated, i.e., a sequence of a few pulses separated from each other by half the driver optical period. While trains of attosecond pulses are comparatively easy to generate, using them for time-resolving processes that extend over more than half an optical period $(\sim 1.3 \mathrm{fs})$ is difficult and requires deconvolution of the overlapping signals from neighboring pulses.
Isolated single attosecond pulses are generated by using short driver pulses where only one optical cycle is strong enough to generate high harmonics at the relevant photon energies [9]. Alternatively, one can also manipulate polarization on the time scale of a single optical cycle in order to suppress high harmonic generation for all but one field peak [10]. Time separation is given by the separation of subsequent driver pulses rather than by the optical halfcycle. These techniques are experimentally complex. While laser oscillators delivering pulses as short as $4 \mathrm{fs}$ FWHM at $80 \mathrm{MHz}$ repetition rate are available [11], amplification to $\gtrsim 10^{14} \mathrm{~W} / \mathrm{cm}^{2}$ brings the repetition rate down into the kilohertz regime.

Plasmonic enhancement was proposed for generating high repetition rate high harmonic pulses directly using laser oscillators [12]. In a recent paper [13], for coneshaped silver funnel geometry, significant field enhancement was theoretically predicted and the detection of extreme ultraviolet (XUV) radiation was reported. In a theoretical study [14], attosecond time structure was found in the response of an isolated atom to the plasmon field.

Here we analyze the macroscopic propagation of the XUV to determine, whether the harmonic radiation is emitted in a usable beam. Our key results are (i) emission from the narrow end of the funnel is severely diffracted rendering it very hard to use in experiments, (ii) in the reverse direction, a well-collimated beam is emitted, (iii) nonlinearity of high harmonic generation ensures a pure attosecond time structure of the emitted pulse, in spite of the temporal distortions of the driver pulse by plasmon dispersion, (iv) harmonics are generated by a single plasmon mode and create a clean spherical wave front, and (v) the source is stable under changes of the driver pulse duration and incidence angle. This "plasmon-enhancedattosecond-XUV source" (PEAX) is compared to standard attosecond gas harmonic sources. While PEAX has significantly lower yield, the unusually clean spatial characteristics and high repetition rates make it an attractive alternative source to standard gas harmonics. 
Our analysis is based on solving the three-dimensional Maxwell equations for propagation of driver, plasmon, and harmonic fields, and solving the atomic time-dependent Schrödinger equation for the microscopic high harmonic response.

Similar to Ref. [13], we chose for our simulations a $9 \mu \mathrm{m}$ long silver cone with an elliptic cross section and opening angles of 14 and 3.5 degrees along the major $(x)$ and minor $(y)$ axis of the ellipse, respectively. We assume a 5 fs FWHM Gaussian driver pulse at wavelength $\lambda_{0}=$ $800 \mathrm{~nm}$, beam waist $w_{0}=2.5 \mu \mathrm{m}$, and focused intensity $I_{0} \approx 4 \times 10^{11} \mathrm{~W} / \mathrm{cm}^{2}$. The focal spot is placed at the larger opening of the cone (see Fig. 1). The field structure can be qualitatively understood by comparing it to the analytic solutions available for the hyperboloid profile [15]: a single $(m=1)$ mode propagates to the tip of the cone, where the peak field is reached. Variations of the focus position by $\pm 1 \mu \mathrm{m}$ cause intensity changes of less than $5 \%$ in peak plasmon intensity. For solving Maxwell's equations, we used the finite difference time-domain open source code MEEP [16]. For algorithmic reasons, dielectric functions in MEEP must have Lorentzian shape, which poorly reproduces the known dielectric response of silver at photon energies around $60 \mathrm{eV}$. Care was taken to accurately fit the imaginary part of the response. We found that the real part of the dielectric response has little influence on the results: with a deliberately bad fit where the real part of the dielectric response was decreased by about a factor of 4 , intensity and time structure of the final harmonic signal change by less than $15 \%$.

As observed in Ref. [13], the elliptic cross section of the cone improves the plasmonic enhancement of the evanescent wave. At the strong eccentricity of $\epsilon=0.25$ we find

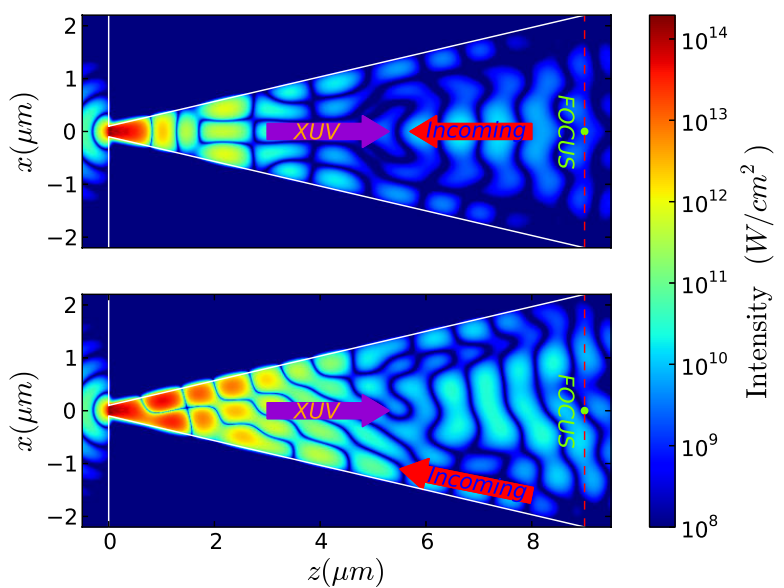

FIG. 1 (color online). Simulation results: intensity distribution in the silver cone in the $x z$ plane at peak plasmon field of $2 \times 10^{14} \mathrm{~W} / \mathrm{cm}^{2}$. Normal (upper panel) and oblique incidence (lower panel). Polarization is in $y$ direction perpendicular to the plane. Oblique incidence angle is 7 degrees in the $z x$ plane parallel to the inner cone surface. In both cases, the harmonic beam is emitted in the direction of the cone axis. an enhancement of $\sim 500$ over the peak intensity of the incident driver field. At $\epsilon=0.5$, used in Ref. [13], the enhancement reduced by about a factor of 3. A similar dependence on ellipticity was reported in Ref. [14], where pulse durations between 4 and 10 fs were investigated. Note that peak field is reached at the surface and therefore surface roughness will introduce modifications of the exact maxima. However, harmonics are produced in volumes of the scale of the evanescent wave, which are less subjected to such subwavelength modifications. Optimizing the cone geometry allows for even weaker driver pulses. However, ultimately the field inside the cone is limited by damage to the silver surface. Fortunately, in Ref. [13] it was found that silver can support much higher fields than expected, likely due to the extreme shortness of the pulses [17].

For high harmonic propagation, the driver field was sampled with grid points separated by $2.5 \mathrm{~nm}$, well below the characteristic wavelength of $800 \mathrm{~nm}$ of the driver plasmon in the cone and below the relevant harmonic wavelengths of $\sim 27 \mathrm{~nm}$. The atomic responses were obtained by solving the time-dependent Schrödinger equation by the infinite range exterior complex scaling method [18] using single-electron models with the ionization potentials of neon, argon, and xenon, respectively. The harmonic yield drops rapidly with driver field intensity: from $2 \times$ $10^{14} \mathrm{~W} / \mathrm{cm}^{2}$ to $1 \times 10^{14} \mathrm{~W} / \mathrm{cm}^{2}$ the yield near the cutoff photon energy of $\sim 60 \mathrm{eV}$ drops by $\sim 3$ orders of magnitude. Therefore, we restricted the calculation of the responses to the "active volume" $V_{a}=x \times y \times z \approx 240 \times 60 \times 500 \mathrm{~nm}^{3}$, where driver intensity exceeded $10^{14} \mathrm{~W} / \mathrm{cm}^{2}$.

Coupling of the driver into the cone and harmonic emission can be maintained over a range of incidence angles. Compared to driver incidence on axis, plasmonic enhancement drops by about a factor of 4 at an incidence angle of 7 degrees around the polarization ( $y$ ) axis, parallel to the inner cone surface at the major axis. The smaller enhancement can be compensated by an increase in driver intensity.

We compute the far field in the three-dimensional geometry from the Kirchhoff integral with the MEEP solution at $z=2.5 \mu \mathrm{m}$. Figure 2 shows the angular distribution of harmonic emission out of the wide side of the cone for a range of harmonic frequencies at oblique driver incidence. The incident intensity was adjusted such as to obtain a peak field in the cone of $2 \times 10^{14} \mathrm{~W} / \mathrm{cm}^{2}$. The beam divergence decreases with increasing photon energy (we define divergence by a decrease to half the peak intensity). For on-axis incidence, similar results are obtained, when the incident intensity is adjusted to give the same peak plasmon intensity. The effect of the driver incidence angle on the emitted beam is small, as the plasmon peak field is dominated by the same single plasmon mode, irrespective of the exact incidence angle.

Waveguiding near the cone tip enhances far-field XUV intensity by a factor of $\sim 4$. Beyond the distance of $2.5 \mu \mathrm{m}$, XUV waveguiding is negligible. We verified this 

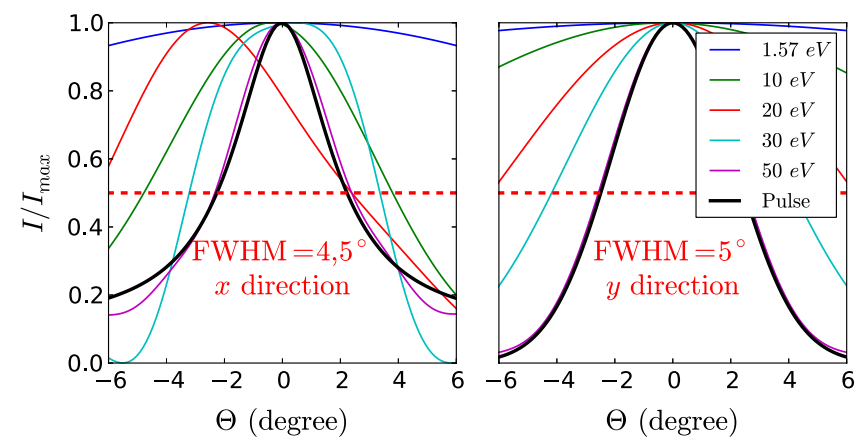

FIG. 2 (color online). Far field angular distribution at a range of photon energies. The asymmetry in the left panel ( $x$ direction) is due to the oblique incidence. The right panel shows polarization $(y)$ direction. Profiles are taken at $1 \mathrm{~mm}$ distance from the cone. The thick black lines show divergence for a pulse composed of all harmonics above $45 \mathrm{eV}$. Thin lines show profile narrowing with increasing photon energy.

by a large distance numerical propagation in a wedgeshaped geometry, where the problem reduces to two dimensions because of translational symmetry.

With its extension $\lesssim 500 \mathrm{~nm}$ the active volume $V_{a}$ where harmonics are generated remains below the driver wavelength. Over that distance, free electron dispersion does not affect phase matching of the harmonic with the dominant plasmon mode. Similarly, atomic dispersion is expected to remain small, and, where needed, may be controlled by choosing a target gas suitable for a given harmonic wavelength. Geometrically induced phase shifts are automatically included in the simulation. With negligible phase slip between driver and harmonic across $V_{a}$, harmonic intensities grow quadratically with the gas density.

The first three columns of Table I shows the parameters of the harmonic pulses obtained for $\mathrm{Ne}, \mathrm{Ar}$, and Xe gases, respectively, at a pressure of 0.3 bar (density $7.8 \times$ $10^{18} \mathrm{~cm}^{-3}$ ), a value typically used for standard gas harmonics. Because of the rapid decay of the spectral intensity with harmonic energy, the central frequency nearly

TABLE I. Harmonic beam characteristics for oblique incidence PEAX for three different gases and a standard harmonic source using a Gaussian beam (see text for parameters). Yields and photon flux are integrated over the beam divergence angles. $V_{a}$ is the active volume.

\begin{tabular}{|c|c|c|c|c|}
\hline \multirow[b]{2}{*}{ Gas } & \multicolumn{3}{|c|}{ PEAX } & \multirow{2}{*}{$\begin{array}{c}\text { Gauss } \\
\text { Ar }\end{array}$} \\
\hline & $\mathrm{Ne}$ & $\mathrm{Ar}$ & $\mathrm{Xe}$ & \\
\hline$\omega_{\gamma}(\mathrm{eV})$ & 53 & 45 & 45 & 45 \\
\hline$\Delta t$ ([as) & 250 & 300 & 250 & 250 \\
\hline Rep. rate & & $80 \mathrm{MHz}$ & & $3 \mathrm{kHz}$ \\
\hline$\gamma /$ pulse & $0.94 \times 10^{-3}$ & $6.7 \times 10^{-3}$ & $0.8 \times 10^{-3}$ & $3 \times 10^{4}$ \\
\hline$\gamma / s$ & $7.6 \times 10^{4}$ & $5.4 \times 10^{5}$ & $6.5 \times 10^{4}$ & $9 \times 10^{7}$ \\
\hline Divergence & & $5^{\circ}$ & & $1^{\circ}$ \\
\hline$V_{a}\left[(\mu \mathrm{m})^{3}\right]$ & & $\sim 4 \times 10^{-3}$ & & $\sim 16$ \\
\hline
\end{tabular}

coincides with the lower cutoff frequency of the harmonics. At a given driver intensity, Ne produces the attosecond pulse at somewhat higher photon energies due to its higher ionization potential. On the other hand, very little ionization $(\mathrm{Ne})$ or very large ionization $(\mathrm{Xe})$ both reduce the achievable yield of high harmonics. This equally affects PEAX and the standard gas harmonic source. The exact electronic structure is of minor importance for the process (see, e.g., Ref. [5]). The PEAX harmonics above $45 \mathrm{eV}$ form an isolated attosecond pulse. Pulse contrast is satisfactory with $85 \%$ of the energy in the central peak.

The harmonic pulse is emitted with a perfectly spherical wave front. At a distance of $1 \mathrm{~mm}$ and over its divergence angle of $5^{\circ}$, the deviation from a spherical shape remains below the central pulse wavelength (Fig. 3). This can be ascribed to the very small and well-defined "focal spot," i.e., the single plasmonic mode where the high harmonics are generated.

The clean wave front allows for focusing without compromising the time structure, which distinguishes this source from standard gas harmonics. The attosecond pulse is emitted on the cone axis, while the reflected driver and lower harmonics are emitted into wider angles with a modulated intensity profile (cf. Fig. 2). This allows simple geometric separation of the incident driver pulse from the harmonic pulse. Remaining on-axis components of the reflected driver and low harmonics can be blocked by standard filters.

The macroscopic silver structure, in addition to supporting the plasmon at the fundamental frequency, also crucially determines XUV emission. Emission through the narrow end of the cone is comparable in photon flux, but beam divergence is $35^{\circ}$ in $y$ direction, such that the narrow end emission is hard to focus for experimental use. The effect is due to diffraction. In a simulation without the guiding and diffraction of the XUV, forward and backward emitted beams are nearly identical.

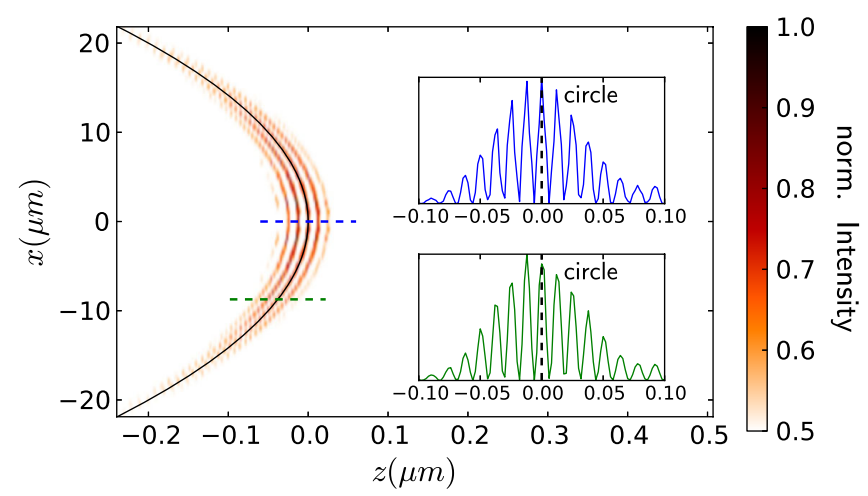

FIG. 3 (color online). Wave front of XUV emission. The insets show cuts through the wave front. The black line indicates a spherical wave front. Dashed lines show position of the cuts and the spherical front, respectively. 
The data given are for a $5 \mathrm{fs}$ driver pulse. A shorter, $4 \mathrm{fs}$ driver bears no further advantage: harmonic pulse duration and divergence remain unchanged, but emitted intensity is reduced by about $20 \%$. Similarly, normal incidence of the driver does not improve harmonic pulse parameters, but rather leads to a reduction of intensity because of the smaller $V_{a}$ (cf. Fig. 1).

In the last column of Table I, the pulse parameters for a traditional harmonic source are listed. For the comparison, a Gaussian beam with 4 fs FWHM pulse duration was used. We assumed a tight focus in a gas jet with peak intensity in focus equal to the peak plasmonic intensity of $2 \times$ $10^{14} \mathrm{~W} / \mathrm{cm}^{2}$ and a beam waist of $1 \mu \mathrm{m}$. At the Rayleigh length of $3.9 \mu \mathrm{m}$, the volume for producing high harmonics is by three orders of magnitude larger than in PEAX. At our specific parameters, PEAX shows slightly longer pulse duration and larger beam divergence. At photon energies $\sim 45 \mathrm{eV}$ and at the given gas pressure, the photon yield per shot is almost 7 orders of magnitude larger compared to PEAX. This ratio corresponds to a ratio of a bit more than 3 orders of magnitude in field strengths, which is consistent with the ratio of the active volumes of the two sources. Because of the low required intensity, driver pulses for PEAX can be directly drawn from a laser oscillator at repetition rates near $100 \mathrm{MHz}$, reducing the ratio of photon fluxes between the two sources to $\lesssim 500$. With the very small $V_{a}$ in PEAX, gas density can be increased significantly before an optical thickness is reached where phase matching problems arise and coherence of the source deteriorates. Assuming tenfold pressure for the PEAX, photon flux could be boosted by 2 orders of magnitude, basically closing the gap to the traditional gas harmonic source. Another obvious extension of PEAX would be to use an array of many instead of a single cone. Only recently, geometries with multiple cones were realized experimentally [19]. In such an arrangement, interference between the emission from different cones could be utilized for phase matching. However, volumes for coherent gas harmonic generation can be significantly larger than what was assumed in our example. Phase matching can be maintained over about $1 \mathrm{~mm}$ propagation length at a beam cross section of a few hundred $\mu \mathrm{m}^{2}$, further increasing volumes by $3-5$ orders of magnitude over our example. This may be a general limit for intensities from any plasmon-enhanced harmonic source: the natural length scale of the high intensity spots in such sources is the driver wavelength, while much larger diameters and phase matching length can be realized in a Gaussian beam.

The present geometry may allow us to experimentally decide, whether reported XUV yields [13] can be ascribed to high harmonic generation or other processes [20]. While the signal in the forward direction must be expected to be severely diffracted, the backward beam may deliver sufficient contrast for proving coherence. Note also that the rough scaling from the cavity source used in Ref. [20] underestimates PEAX yields from the present simulation by several orders of magnitude.

The advantages of PEAX are its submicrometer collimated attosecond XUV beam, which may be used for spatiotime-resolved surface spectroscopy [8], and the high repetition rates that reduce space charge effects on the target. In principle, with the high repetition rate and possibly few-cycle pulses in the $45 \mathrm{eV}(\sim 27 \mathrm{~nm}$ wavelength) regime, frequency combs could be pushed to new parameter regimes. As plasmonic fields may vary over very small spatial ranges, nondipole modifications of the atomfield interactions are introduced that can be exploited, e.g., for further reduction of pulse duration and for higher photon energies [21].

We acknowledge support by the DFG, by the excellence cluster "Munich Center for Advanced Photonics (MAP)", and by the Austrian Science Foundation Project ViCoM (F41). M. L. was supported by IMPRS "Advanced Photon Science." M. F. K. acknowledges personal support by U.S. Department of Energy under Contract No. DE-SC008146 and the DFG via Contract No. Kl-1439/5. We are grateful for discussions with V. Yakovlev.

*mattia.lupetti@physik.uni-muenchen.de

†armin.scrinzi@1mu.de

[1] M. Drescher, M. Hentschel, R. Kienberger, M. Uiberacker, V. Yakovlev, A. Scrinzi, Th. Westerwalbesloh, U. Kleineberg, U. Heinzmann, and F. Krausz, Nature (London) 419, 803 (2002).

[2] T. Remetter et al., Nat. Phys. 2, 323 (2006).

[3] A.L. Cavalieri et al., Nature (London) 449, 1029 (2007).

[4] E. Goulielmakis et al., Nature (London) 466, 739 (2010).

[5] P. B. Corkum, Phys. Rev. Lett. 71, 1994 (1993).

[6] K.C. Kulander, K. J. Schafer, and J.L. Krause, in Proceedings of the Workshop Super Intense Laser Atom Physics (SILAP) III, edited by B. Piraux (Plenum, New York, 1993).

[7] K. Zhao, Q. Zhang, M. Chini, Y. Wu, X. Wang, and Z. Chang, Opt. Lett. 37, 3891 (2012).

[8] M. I. Stockman, M. F. Kling, U. Kleineberg, and F. Krausz, Nat. Photonics 1, 539 (2007).

[9] A. Baltuška et al., Nature (London) 421, 611 (2003).

[10] I. Sola et al., Nat. Phys. 2, 319 (2006).

[11] S. Rausch, T. Binhammer, A. Harth, J. Kim, R. Ell, F. X. Kärtner, and U. Morgner, Opt. Express 16, 9739 (2008).

[12] S. Kim, J. Jin, Y.-J. Kim, I.-Y. Park, Y. Kim, and S.-W. Kim, Nature (London) 453, 757 (2008).

[13] I.-Y. Park, S. Kim, J. Choi, D.-H. Lee, Y.-J. Kim, M. F. Kling, M. I. Stockman, and S.-W. Kim, Nat. Photonics 5, 677 (2011).

[14] J. Choi, S. Kim, I.-Y. Park, D.-H. Lee, S. Han, and S.-W. Kim, New J. Phys. 14, 103038 (2012).

[15] A. Passian, R. Ritchie, A. Lereu, T. Thundat, and T. Ferrell, Phys. Rev. B 71, 115425 (2005). 
[16] A. F. Oskooi, D. Roundy, M. Ibanescu, P. Bermel, J. D. Joannopoulos, and S. G. Johnson, Comput. Phys. Commun. 181, 687 (2010).

[17] A. Plech, V. Kotaidis, M. Lorenc, and J. Boneberg, Nat. Phys. 2, 44 (2006).

[18] A. Scrinzi, Phys. Rev. A 81, 053845 (2010).
[19] I.-Y. Park, J. Choi, D.-H. Lee, S. Han, S. Kim, and S.-W. Kim, Ann. Phys. (Berlin) 525, 87 (2013).

[20] M. R. Raschke, Ann. Phys. (Berlin) 525, A40 (2013).

[21] T. Shaaran, M. F. Ciappina, and M. Lewenstein, Phys. Rev. A 86, 023408 (2012). 